\title{
Non-revisiting Coverage Task with Minimal Discontinuities for Non-redundant Manipulators
}

\author{
Tong Yang, Jaime Valls Miro, Yue Wang* and Rong Xiong
}

\begin{abstract}
A theoretically complete solution to the optimal Non-revisiting Coverage Path Planning (NCPP) problem of any arbitrarily-shaped object with a non-redundant manipulator is proposed in this work. Given topological graphs of surface cells corresponding to feasible and continuous manipulator configurations, the scheme is aimed at ensuring optimality with respect to the number of surface discontinuities, and extends the existing provable solution attained for simply-connected configuration cell topologies to any arbitrary shape. This is typically classified through their genus, or the number of "holes" which appear increasingly as configurations are further constrained with the introduction of additional metrics for the task at hand, e.g. manipulability thresholds, clearance from obstacles, end-effector orientations, tooling force/torque magnitudes, etc.

The novel contribution of this paper is to show that no matter what the resulting topological shapes from such quality cell constraints may be, the graph is finitely solvable, and a multistage iterative solver is designed to find all such optimal solutions.
\end{abstract}

\section{INTRODUCTION}

Full coverage of the surface of a given object with nonredundant manipulators is embodied in tasks such as automatic polishing, deburring, painting or surface inspection, where the need for non-repetitive path planning is paramount to avoid over revisiting.

The kinematic relationship of a typical manipulator makes mapping between work- and joint-space non-bijective [11], which in effect drives coverage paths to be traditionally carried out in the former to ensure no revisiting of points in the surface [15]. However, in further pursuing motions where the manipulator may minimise the number of reconfigurations is obliged to undertake to follow a desirable continous endeffector (EE) path, a global optimal cellular decomposition problem in joint-space has been proposed to incur joint-space partitions with minimum sets [20]. This is illustrated in the example shown by Fig. 11 the external surface of a wok-like object is inspected by a non-redundant manipulator. Points on the surface can be reached by a variety of robot configurations. The three solid colour cells shown in Fig. 1(b) illustrate poses of disjoint sets reachable as a continous set by a given configuration, visually seen by the different colours. These cells become the elements on a topological graph Fig. 1(c), where each possible colour of a cell is recorded. A cellular decomposition splits and merges the cells to transform the

\footnotetext{
${ }^{1}$ Tong Yang, Yue Wang and Rong Xiong are with the State Key Laboratory of Industrial Control and Technology, Zhejiang University, P.R. China.

2 Jaime Valls Miro is with the Centre for Autonomous Systems (CAS), University of Technology Sydney (UTS), Sydney, Australia.

* Corresponding Author.

E-mail address: wangyuediipc.zju.edu.cn
}

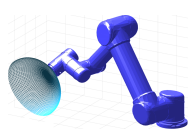

(a)

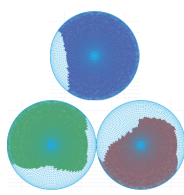

(b)

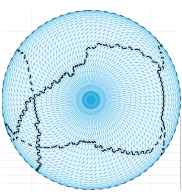

(c)

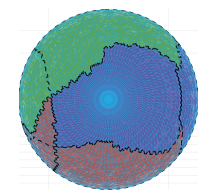

(d)
Fig. 1. Example of simply-connected cell coverage. (a) Hemispherical object placed in the workspace. (b) Simply-connected cells of three valid robot configurations, chosen by the optimal solution shown in (d). (c) Topological graph. (d) One optimal solution requiring 2 lift-offs.

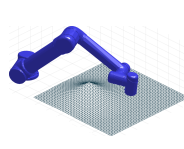

(a)

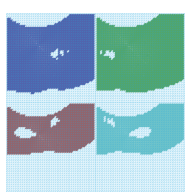

(b)

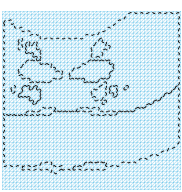

(c)

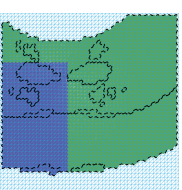

(d)
Fig. 2. Example of multiply-connected cell coverage. (a) Irregular "hilly" object placed in the workspace. (b) Multiply-connected cells of four valid robot configurations, chosen by the optimal solution shown in (d). (c) Topological graph. (d) One optimal solution requiring 1 lift-off.

process into that of painting all points in the graph with one of the possible colours, driven by attaining a minimum set which equates to least number of EE lift-offs. One such optimal solution with 2 lift-offs is illustrated in Fig. 1(d), whereby any arbitrary continuous path within a colour surface area will result in maxium joint continuity of the global path. The addition of obstacles and the relative pose of robot and object further condition the final solution. For further details please refer to [20].

A critical assumption however is that the constituent surface points necessarily adopt simply-connected space topologies. This is reasonable for simple shapes and sparse obstacle environments, but becomes unrealistic as objects grow in complexity, or with the imposition of increasing motion constraints, generically deriving in multiply-connected cells. An illustrative example is given in Fig. 2 where a manipulator moving over a "hilly" terrain generates reachable areas in the mesh which contains non simply-connected cells. This is further compounded when additional or more restrictive constraints are imposed, as will be seen later in the results when the degree of manipulability is also considered and more "cavities" appear in the configuration cells and generated topological graphs.

Since the shape of cells can be classified through their genus, i.e., the number of "holes" within the cell, in this work a theoretically complete solution is given by solving for cells 
with all possible genus. The key observation of the proposed algorithm is that whatever genus the cell is, the number of liftoff to cover it is always zero. A direct reflection from this is thus to reduce the genus of the cell gradually by cell divisions to eventually make it equivalent to a simply-connected cell which has been proven finitely solvable. This however is non-trivial: the place to put a cutting path to transform a genus-one cell into a simply-connected cell directly effects the order of edges on the boundary of the sub-cells, leading to different structures. Existing algorithms in the literature are not able to provide a systematic cell decomposition solution to accomplish a guaranteed transformation from an initial graph containing large genus cells into simply connected topologies. This is provided in this work through the analysis of equivalent topological divisions. To that extent, the finiteness of all possible divisions is first proven for a genus-one cell as building block. A multi-stage iterative solver can then be introduced to transform higher-genus cells into lower-genus cells. The two novel contributions of this paper can be thus summarised as:

1) Providing the necessary conditions to be able to apply the surface modeling described by the proposed topological graph structure.

2) Proposing a systematic solution to be applicable for any genus- $n$ cell topology.

The remainder of this paper 1 is organised as follows. Section II reviews existing literature. Section IV proves the necessary conditions to apply the proposed algorithm. Section $\mathrm{V}$ briefly restates the existing results in [20] to solve for simply-connected cells. Section [VI goes into further details about the finiteness of solving elementary genus-one cells, whilst a multi-stage iterative strategy to solve for larger genus$n$ cells is described in Section VII Experimental results from simulations are collected in Section VIII, with final concluding remarks gathered in Section IX.

\section{RELATED WORKS}

Almost all state-of-the-art methods to solve the coverage path planning (CPP) problem [5] [7] first divide the target surface into cells then solve the CPP problem in each cell, so called cellular decomposition, which is further divided into two categories: exact cellular decomposition methods [13] and Morse-based cellular decomposition methods [6] [1].

For the optimal coverage task with a manipulator, the algorithms mainly focus on workspace metrics such as path length and time to completion. Atkar et al. [2] optimised the coverage path through choosing optimal starting points. Huang [10] reduced movement cost by remaining on straight paths as long as possible thus minimising the number of turns. In dealing with the optimal Non-revisiting Coverage Path Planning (NCPP) problem, [3] considered the uniform coverage in automotive spray painting problem, where the simple backand-force boustrophedon path was deformed in accordance to the curvature and topology of the surface. [8] proposed

${ }^{1}$ A video illustrating the concepts hereby described can be found here https: //youtu.be/TqFzqGGM06Y a 3D coverage path for agricultural robots minimising the skip/overlap areas between swaths.

Its is arguable however that for surface contact tasks in particular, the cost incurred in joint discontinuities significantly outweighs other metrics, moreover where undesirable transitions between position and force/torque control [4] [9] [14] [18] [19] become unavoidable.

We notice that [16] considered the pose optimisation of a mobile manipulator for coverage task, where a quality measurement was proposed to optimally place the manipulator in the workspace to aid coverage. The focus remained complementing robot placement and CPP; the actual generation of the coverage path simply reduced to use a randomised path planner, BiRRT [12] among a set of "guard points" chosen, with no specfic consideration to joint-space continuity as ellaborated in the present manuscript.

It is worthwhile noting that despite the apparent similarities in planning with mobile platforms, the optimal NCPP problem with least discontinuities is inherent to the kinematics of manipulator mechanisms, and as such beyond the scope of bibliograpic works from the mobile robotics community. Likewise, affinities to graph theory are also legit. Note however that the contact point, which can be safely regarded as a particle, is significantly smaller than the scale of the cellular decomposition drawn up for this problem. In seeking maximal jointspace continuity of the coverage path travelled by a particle as modelled by the NCPP problem, infinitesimal elements must be considered in its most abstract form, and the existance of infinitely narrow passages also makes a significant difference in the resulting path solutions. Hence, it is not possible to revert back to classical graph theory since no any area on the surface can be seen as a whole to form the "vertices", and the set of "edges" is actualy the exact solution the NCPP problem is seeking to solve for.

\section{Problem Statement}

Define the surface of an object by $M$. To simplify the description, let $M$ be only the coverable part of the surface. The shape of other obstacles in the workspace and their relative poses in the workcell are assumed static and known. Given the kinematics of the non-redundant manipulator, we denote the joint-space by $\overline{\mathscr{C}}$, and the set of all singular configurations by $S$. The set of all valid manipulator configurations is denoted by $\mathscr{C}$, where the validity of a configuration is evaluated by some given quality constraints denoted by $\left\{F_{k}\right\}_{k \in \mathbb{N}}$. Classic examples would be manipulability or the minimum distance from an obstacle. The optimal CPP problem is to find a joint-space path consisting of valid configurations whereby the manipulator EE covers the workspace non-repetitively and ensures the least number of discontinuities requiring lifting from the object's surface. The following settings define the scope formally:

1) A point contact between surface and EE is assumed.

2) Let $s \in S$ be a singular configuration, there exist a threshold $\varepsilon>0$ such that

$$
d(c, s)>\varepsilon, \forall c \in \mathscr{C}, \forall s \in S
$$


where $d(\cdot, \cdot)$ is a metric in the joint-space. This enables us to judge the discontinuity of two configurations coming from discretised data. For example, given two configurations $c_{1}, c_{2} \in \mathscr{C}$ corresponding to the EE covering two adjacent vertices of a triangular mesh, we say $c_{1}$ and $c_{2}$ are joint-space continuous only if

$$
d\left(c_{1}, c_{2}\right)<\varepsilon
$$

where $d(\cdot, \cdot)$ and $\varepsilon$ are pre-determined.

3) $\left\{F_{k}\right\}_{k \in \mathbb{N}}$ is continuously defined in the joint-space, with thresholds satisfying strict inequalities. The $k$-th constraint can be expressed as

$$
F_{k}(c)>\delta_{k}=\delta_{k}\left(c, F_{1}, \cdots, \hat{F}_{k}, \cdots, F_{n}\right), \forall c \in \mathscr{C}
$$

where "^" means excluding the term. $\delta_{k}$ can be a function of other metrics instead of a constant value, leading to wider applications. For example, let $F_{1}$ represent the manipulability [21] and $F_{2}$ represent the minimum distance between the manipulator and all obstacles, then an inequality such as

$$
F_{2}(c)>\delta_{2}=0.01+0.05\left(1-F_{1}(c)\right)
$$

could be imposed to indicate that when $F_{1}(c)$ is small, i.e. a "badly-conditioned" configuration, it should be farther from obstacles than would be desirable otherwise.

\section{Finiteness of CELlS}

For a non-redundant manipulator, $\forall m \in M$ there exist a finite number of valid configurations to cover $m$. By following the constraints laid out in the problem statement, a topological graph will be generated. A necessary condition to solve it is thus guaranteeing the finitness in the number of cells on this graph. For that, let us define a topological cell $C_{1}$ (where 1 represents its index) as a maximal set of continuous points where the valid configurations to cover them are pairwise continuous, i.e., $\forall m, m^{\prime} \in C_{1}$, let the valid configurations to cover them be

$$
\begin{gathered}
P_{m}=\left\{c_{m 1}, \cdots, c_{m N}\right\} \subset \mathscr{C} \\
P_{m^{\prime}}=\left\{c_{m^{\prime} 1}, \cdots, c_{m^{\prime} N^{\prime}}\right\} \subset \mathscr{C}
\end{gathered}
$$

then $N=N^{\prime}$ and $\forall c_{m i} \in P_{m}, \exists c_{m^{\prime} j} \in P_{m^{\prime}}, c_{m i}$ and $c_{m^{\prime} j}$ are jointspace continuous.

A topological edge $E_{1}$ (where 1 represents its index) is a maximal set of continuous points between $C_{i}$ and $C_{j}$ whereby

$$
\begin{aligned}
E_{1} & =E_{1}\left(C_{i}, C_{j}\right) \\
& =\left\{m \in M \mid \forall(m \in) U_{m}, \exists p, p^{\prime} \in U_{m}, p \in C_{i}, p^{\prime} \in C_{j}\right\}
\end{aligned}
$$

where $U_{m}$ is an open set containing $m$. Note that the intersecting points of topological edges

$$
\left\{m \mid \forall(m \in) U_{m}, \exists p, p^{\prime}, p^{\prime \prime} \in U_{m}, p \in C_{i}, p^{\prime} \in C_{j}, p^{\prime \prime} \in C_{k}\right\}
$$

are distinct points, which is negligible when discussing the connectivity of the cells. The topological graph is the ordered cells and edges, $G=\left(\left\{C_{i}\right\},\left\{E_{j}\right\}\right)$.
To prove the finitness of this graph, let us randomly choose a point $m \in M$ which can be covered by a set of valid configurations $P_{m}$ defined by (4), then

$$
F_{k}\left(c_{m i}\right)>\delta_{k}, \forall k, \forall i=1, \cdots, N
$$

the strict inequalities (3) enable us to find valid open sets $U_{m i}$ for each configuration $c_{m i}$ in the joint-space:

$$
\begin{gathered}
\exists\left(c_{m i} \in\right) U_{m i} \subset \mathscr{C}, \forall i=1, \cdots, N \\
\text { s.t. } F_{k}(c)>\delta_{k}+\frac{1}{2}\left(F_{k}\left(c_{m i}\right)-\delta_{k}\right)>\delta_{k}, \forall k, \forall c \in U_{m i}
\end{gathered}
$$

since the kinematic function of the manipulator $(\mathrm{FK})$ is continuous and $c_{m i} \in U_{m i}$,

$$
m \in \operatorname{FK}\left(U_{m i}\right) \subset M, \forall i=1, \cdots, N
$$

so does the intersecting area of those images,

$$
m \in A_{m} \triangleq \bigcap_{i=1}^{N}\left(\mathrm{FK}\left(U_{m i}\right)\right) \subset M, \forall i=1, \cdots, N
$$

follow the definition of our topological cells, all points within the intersecting area belong to a same cell.

For $\forall m \in M$, the corresponding open set $A_{m}$ exists, as such

$$
\bigcup_{m \in M} A_{m} \supseteq M
$$

hence the left term forms a infinite cover of $M$. The HeineBorel Theorem [17] states that if a compact region can be covered by infinite many open regions, then one can find finite many of them that still fully cover this region. Since all closed sets in the Euclidean space are compact sets, so is the manipulator task space in which the boundaries are welldefined. Thus, we can find finite elements from $\left\{A_{m}\right\}_{m \in M}$ that also fully cover $M$. Recall that each $A_{m}$ belongs to only one cell, hence the total number of cells in the graph must be finite.

\section{Solving GRaphs with Simply-Connected Cells}

In earlier work [20], the optimal NCPP problem with least discontinuities was also modelled as a topological cell graph, and the solution transformed into an optimal design strategy of a colour (configuration) scheme whereby the strategic placement of cutting paths would invariably lead to different colour vertices on both sides of a partition. An illustrative example is provided by Fig 1. In proving the finiteness of simply-connected cells, the following claims were validated:

1) It is sufficient to consider cutting paths that start and end at the topological edge end-points.

2) It is unnecessary to consider cutting paths that stretch across edges.

3) Intersecting cutting paths can be discarded.

4) For a simply-connected cell with $K$ topological edges delineating its boundary, a binary number of length $K$ can uniquely specify the set of all admitted divisions.

5) To solve a simply-connected cell with $K$ edges, the total number of different divisions $\Phi$ is bound by

$$
\Phi(K) \leq 2^{K}
$$

In the following sections, a generalisation to genus- $n$ cells is derived. 


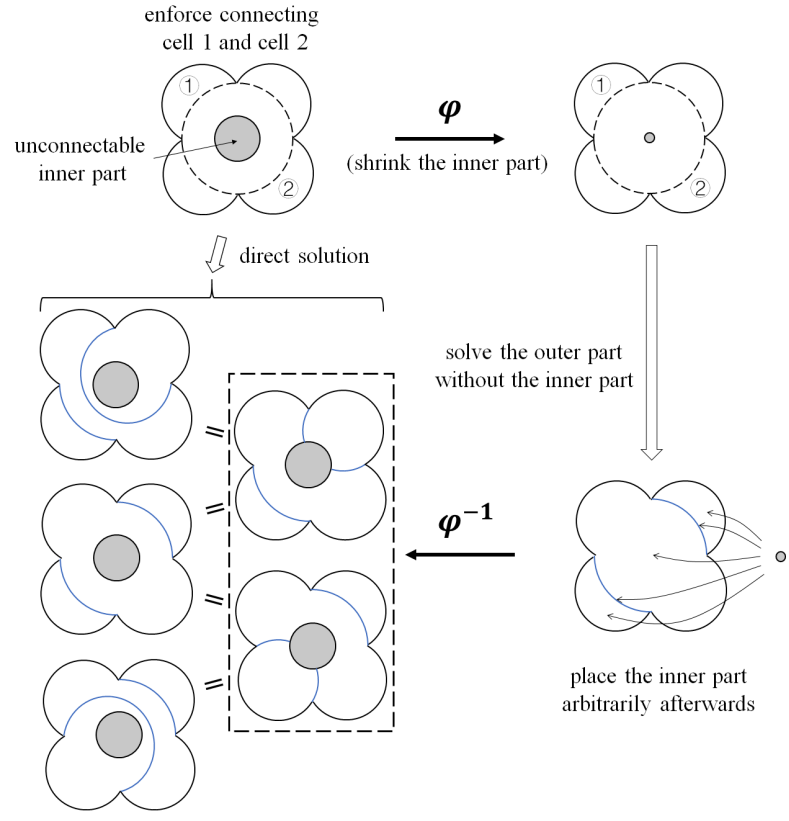

Fig. 3. Example showing that the unconnectable inner part (marked by the grey solid circle) is negligible when solving the outer part. $\omega$ and $\omega^{\prime}$ are directly drawn as concentric circles so $\varphi\left(\omega^{\prime}\right)=\omega^{\prime}$. In this simple example the direct solutions are straightforward, and they are equivalent to first solving the outer part without the inner part, and then arbitrarily replacing back the inner part. It can also be observed how the inner part can be placed "on" a cutting path (dashed block), yet there is no need not consider these cases since they are effectively equivalent to the three divisions shown on the left under a continuous cutting path transformation.

\section{Solution of Genus-One Cells}

A cutting path connecting the inner part and the outer part of a genus-one cell will transform the cell into a simplyconnected cell, proven to be finitely solvable. In this section we consider two cases: keeping the genus-one structure or transforming the genus-one cell into simply-connected subcells. In the former case, we prove that the inner part and the outer part can be seen as two independent problems. While in the later case, by efficiently discarding equivalent cellular decompositions, the number of possible subdivisions is proven to be finite, and all optimal solutions thus finitely solvable. An upper-bound on the complexity of solving a genus-one cell is also derived.

For notation, a genus-one cell is denoted by $\Omega$, with its inner boundary and outer boundary denoted by $\omega$ and $\omega^{\prime}$, respectively. The edges and cutting paths are denoted by $\alpha$.

\section{A. Independence of Inner and Outer Parts}

The situation when inner part and the outer part of $\Omega$ are not connected arises when the inner part is a physical hole, the inner part can only be covered with a totally different colour from that of the outer part, or when all the edges in $\omega$ must be retained. In these cases, first we prove that the inner part of $\Omega$ is negligible when solving the outer part. To show this equivalence, let $\varphi$ be a homeomorphic mapping from $\Omega$ to the standard unit disk $D$, with its boundaries mapped onto concentric circles,

$$
\varphi: \Omega \rightarrow D, \omega^{\prime} \mapsto S^{1}(1), \omega \mapsto S^{1}(\delta), \delta \rightarrow 0^{+}
$$

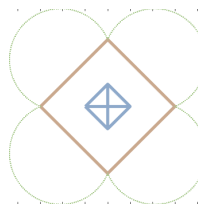

(a)

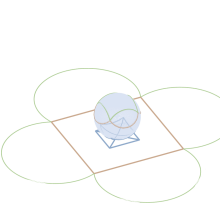

(b)

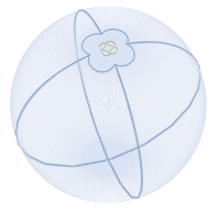

(c)
Fig. 4. (a) Topological graph with a genus-one cell enforced to be kept (the area between concentric squares). (b) A stereographics projection using a sphere lying within the inner boundary. (c) Let the radius of the sphere be small enough, then the inner part of graph and the outer part interchange near the north pole of the sphere.

then the inner part becomes an infinitesimal open neighbourhood of the origin. See Fig. 3 for illustration. Let a cutting path of $D, \varphi(\alpha)$, start and end at $\varphi\left(\omega^{\prime}\right)$, then it must be one of the following two cases:

$$
\begin{aligned}
& (0,0) \notin \varphi(\alpha) \\
\Rightarrow & \exists \varepsilon>0, B(0, \varepsilon) \cap \varphi(\alpha)=\varnothing \\
\Rightarrow & \text { let } \delta<\varepsilon, \text { then } \varphi(\omega) \cap \varphi(\alpha)=\varnothing \\
\Rightarrow & \omega \cap \alpha=\varnothing
\end{aligned}
$$

or

$$
\begin{aligned}
& (0,0) \in \varphi(\alpha) \\
\Rightarrow & \varphi(\omega) \cap \varphi(\alpha) \neq \varnothing, \forall \delta \\
\Rightarrow & \omega \cap \alpha \neq \varnothing
\end{aligned}
$$

All the different topological divisions described by and (16) correspond to those listed in Fig. 3 .

Next, we prove the inverse claim that the outer part of $\Omega$ is negligible when solving the inner part. Let a sphere with radius $r$ stand within the inner part (anywhere is applicable but a place within the inner part will simplify the description), and $\psi$ be the stereographic projection

$$
\begin{aligned}
\psi: \mathbb{R}^{2} & \rightarrow S^{2} \backslash N \\
(x, y, 0) & \mapsto\left(\frac{4 r^{2} x}{x^{2}+y^{2}+4 r^{2}}, \frac{4 r^{2} y}{x^{2}+y^{2}+4 r^{2}}, \frac{2 r\left(x^{2}+y^{2}\right)}{x^{2}+y^{2}+4 r^{2}}\right)
\end{aligned}
$$

where $N$ denotes the arctic point. See Fig. 4 for illustration. Since $\psi$ is homeomorphic, the transformed graph on the sphere is equivalent to the original graph. $\psi$ maps the outer part (including the uncoverable ambient space) to a neighbourhood of the arctic point (the $\infty$ point to the arctic point), and the inner part to a neighbourhood of the antarctic point. Let $r \rightarrow 0$, the roles of the inner part and the outer part interchange near the arctic point. Recalling the observation of the negligibility of the inner part, we finish the proof.

In summary, when the inner part and the outer part are not connected, they can be solved independently and their results can be merged directly.

\section{B. Negligibility of Cyclic and Single Cutting Paths}

Cutting paths connecting $\omega$ and $\omega^{\prime}$ can be classified based on their number of cycles, as depicted by Fig. 5(a). However, Fig. 5(b) shows that all cyclic cutting paths have the same topological function as the acyclic one, thus can be safely disregarded. Note however that unlike the case for other equivalences, the physical cellular decomposition using the 


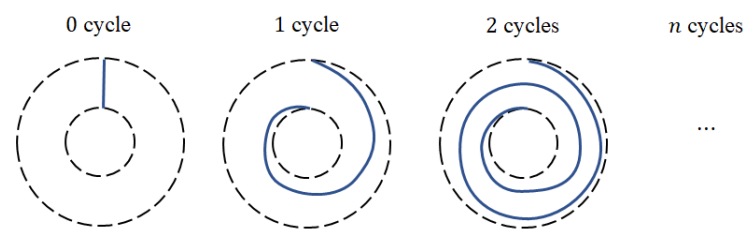

(a) Each acyclic cutting path corresponds to a family of cyclic cutting paths that go through different cycles.

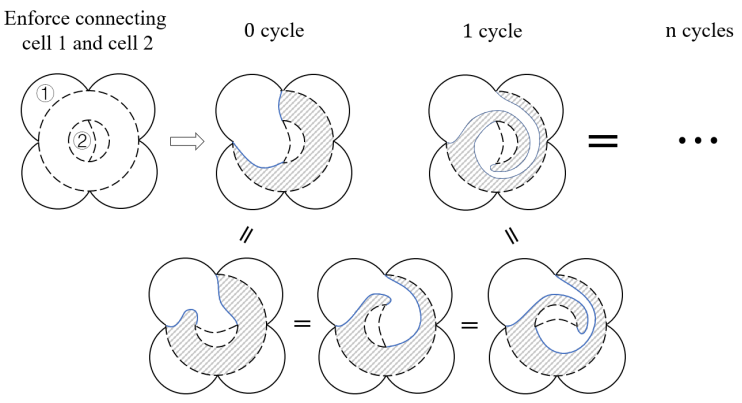

(b) Continuous transformation between the 0-cycle division and 1-cycle division, with similar results for the $n$-cycles case.

Fig. 5. Examples of negligible cutting paths.

cyclic cutting paths cannot be generated through continuously modifying the position of the acyclic ones. Here the equivalence means that only the acyclic situations require explicit consideration. Once an acyclic solution is optimal, all equivalent solutions using the cyclic cutting paths are all optimal and automatically considered.

Next, we observe that if there is only one cutting path that connects $\omega$ and $\omega^{\prime}$, then $\Omega$ is transformed into a single simplyconnected sub-cell. However, this becomes a contradiction since both sides of the cutting paths are effectively the same cell, thus impossible to have different colors. It is therefore apparent that it is not the cutting path but the connected area what determines the division of $\Omega$. More precisely, an area connecting the inner part and the outer part of $\Omega$. This observation motivates us to directly consider the connectivities between cells. Based on the remarks in Section V, we only need to judge whether keeping or removing each edge. Let the indices of $J$ edges in $\omega$ and $K$ edges in $\omega^{\prime}$ be (in cyclic order)

$$
\begin{aligned}
\omega & =\left(\alpha_{1}, \cdots, \alpha_{J}\right) \\
\omega^{\prime} & =\left(\alpha_{1}^{\prime}, \cdots, \alpha_{K}^{\prime}\right) .
\end{aligned}
$$

Arbitrarily choosing some edges in $\omega$ and $\omega^{\prime}$ to generate a connectivity, like

$$
\left(\left\{\alpha_{i_{1}}, \cdots, \alpha_{i_{r}}\right\},\left\{\alpha_{j_{1}}^{\prime}, \cdots, \alpha_{j_{s}}^{\prime}\right\}\right), 1 \leq r \leq J, 1 \leq s \leq K
$$

where \{\} means the order has no meaning, leads to valid divisions of the $\Omega$. An example is shown in Fig. 6, where it is apparent that results are not unique. The finiteness of this approach is discussed next.

\section{Assignment of Cutting Paths Based on Connectivity}

Extracting all combinations from (20) does not necessarily mean listing all different divisions. In Fig. 6, various divisions

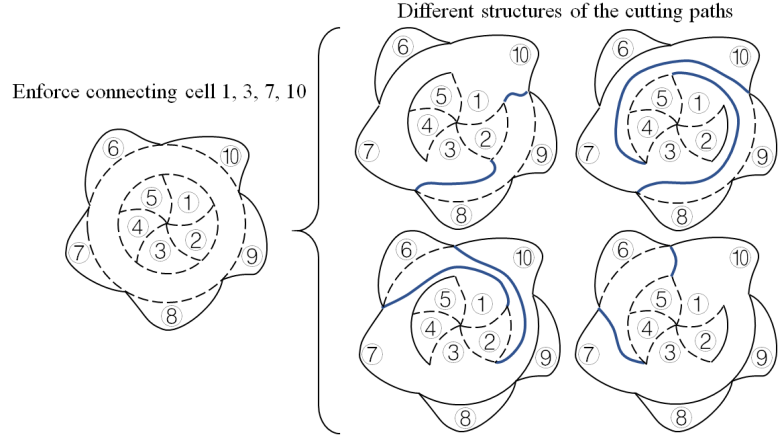

Fig. 6. In this example, cells 1, 3, 7 and 10 are enforced to be connected. The four distinct divisions shown on the right are all valid, exemplifying the non-uniqueness of the subdivision problem.

are created from the same connectivity. In order to address this issue, the whole division of $\Omega$ is regarded as a two-stage process, first transforming into simply-connected sub-cells and then undertaking further divisions in these sub-cells. We prove that in the first stage, only two cutting paths are required which connect $\omega$ and $\omega^{\prime}$.

First, all cutting paths that do not connect $\omega$ and $\omega^{\prime}$ do not appear in the first stage, because they can be seen as further divisions in the generated sub-cells. Refer to Fig. 7 for an illustration of this phenomenon. Then, let there be three cutting paths that connect $\omega$ and $\omega^{\prime}$, then $\Omega$ is divided into three simply-connected cells. However, the resulting topological structure is equivalent to $\Omega$ being firstly divided into two sub-cells, then another cutting path creating further divisions iteratively. Hence, there need to be at most two cutting paths in the first-stage.

In summary, let $\omega$ and $\omega^{\prime}$ be defined by (18) and (19), during the first stage a continuous subset is identified in $\omega$, and another one in $\omega^{\prime}$. The total number of different divisions is the number of different choices of these continuous subsets. To relieve the problem of the cyclic choices, we enforce the sub-cell 1 be the one containing $\alpha_{1}$. So all choices in $\omega$ are described by

$$
\left\{\begin{array}{l}
\left\{\alpha_{1}\right\} \\
\left\{\alpha_{1}, \alpha_{2}\right\},\left\{\alpha_{J}, \alpha_{1}\right\} \\
\cdots \\
\left\{\alpha_{1}, \cdots, \alpha_{J-1}\right\}, \cdots,\left\{\alpha_{3}, \cdots, \alpha_{J}, \alpha_{1}\right\} .
\end{array}\right.
$$

Likewise, all choices in $\omega^{\prime}$ are given by

$$
\left\{\begin{array}{l}
\left\{\alpha_{1}^{\prime}\right\},\left\{\alpha_{2}^{\prime}\right\}, \cdots,\left\{\alpha_{K}^{\prime}\right\} \\
\left\{\alpha_{1}^{\prime}, \alpha_{2}^{\prime}\right\}, \cdots,\left\{\alpha_{K}^{\prime}, \alpha_{1}^{\prime}\right\} \\
\cdots \\
\left\{\alpha_{1}^{\prime}, \cdots, \alpha_{K-1}^{\prime}\right\},\left\{\alpha_{K}^{\prime}, \alpha_{1}^{\prime}, \cdots, \alpha_{K-2}^{\prime}\right\}, \cdots,\left\{\alpha_{2}^{\prime}, \cdots, \alpha_{K}^{\prime}\right\}
\end{array}\right.
$$

\section{Complexity Analysis}

When $\omega$ and $\omega^{\prime}$ are connected, there are $r$ methods to choose $r$ continuous edges in $\omega$ containing $\alpha_{1}$, i.e.,

$$
\left\{\alpha_{J-r+1}, \cdots, \alpha_{J}, \alpha_{1}\right\}, \cdots,\left\{\alpha_{1}, \cdots, \alpha_{r}\right\}
$$




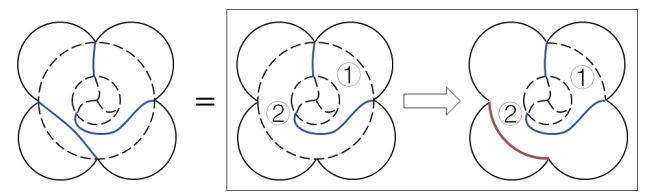

(a) Illustration of cutting paths that start and end at the same boundary, which can be seen as further divisions (in sub-cell 2, shown in red).

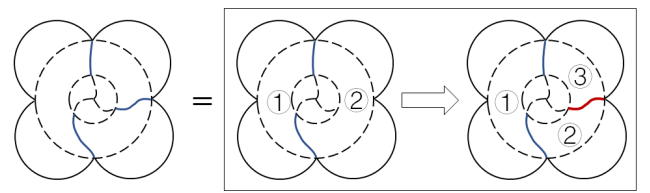

(b) Example shows that the third (and later) cutting paths connecting $\omega$ and $\omega^{\prime}$ can be seen as further divisions (in sub-cell 2, shown in red).

Fig. 7. The placement of cutting paths can be seen as a two-stage processes: First we only place two cutting paths that connect the inner boundary and the outer boundary, and then place all other cutting paths within the simplyconnected sub-cells, seen as further divisions of the sub-cells.

and $K$ methods to choose $s$ continuous edges in $\omega^{\prime}$. Summarising all possible $r$ and $s$ we get

$$
\Psi(J, K)=\sum_{r=1}^{J-1} \sum_{s=1}^{K-1} r K \Phi(r+s) \Phi(J+K-r-s)
$$

with $\Phi$ defined by (13). Moreover, from Section VI-A, the number of different divisions keeping a genus-one structure is

$$
\Phi_{0}(J, K)=\Phi(J)+\Phi(K)=2^{J}+2^{K}
$$

hence the total number of different divisions for a genus-one cell with $J$ edges in the inner boundary and $K$ edges in the outer boundary is given by

$$
\Phi(J, K)=\Phi_{0}(J, K)+\Psi(J, K)
$$

\section{Solution of LARGe Genus Cells}

There are $n$ inner boundaries in a genus- $n$ cell. Taking the outer boundary into consideration, there are $C_{n+1}^{2}$ possible "starting- ending-point" pairs for the possible cutting paths, which are impractical to enumerate and deal with. Hence, a multi-stage iterative strategy is once again proposed to transform a genus- $n$ cell into sub-cells with genus no more than $n-1$.

\section{A. Genus-Two Cell Case}

Following a deduction process similar to the one in Section VI-A, the ensuing equivalence states arise:

1) The disconnected inner part can be safely discarded and the remaining becomes a genus-one cell which has been proved solvable. After solving both the genus-one cell and the unconnectable part independently, we can arbitrarily place the resulting division of the disconnected inner part in the result of the genus-one cell.

2) When both inner parts are unconnectable, the genus-two cell can be seen as three simply-connected parts. And the solution comes from the arbitrary placements of the solution of the inner parts in the result of the outer part.

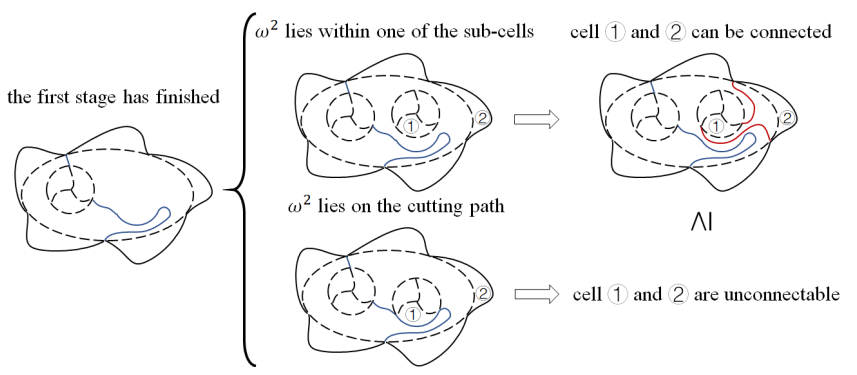

Fig. 8. Example showing that at each stage, cost-wise it is unnecessary to place the second inner part on the cutting paths created earlier (in blue).

For a genus- $n$ cell, if any of its inner part is disconnected, the genus- $n$ problem becomes a genus- $(n-1)$ problem which is proven solvable under induction. Hence, in this section the focus is on the case when all boundaries are forced to be connected.

Following the notation of a genus-one cell, a genus-two cell is denoted by $\Omega$, with its outer boundary described by

$$
\omega=\left(\alpha_{1}, \cdots, \alpha_{K}\right)
$$

and the inner boundaries given by

$$
\begin{aligned}
& \omega^{1}=\left(\alpha_{1}^{1}, \cdots, \alpha_{J_{1}}^{1}\right) \\
& \omega^{2}=\left(\alpha_{1}^{2}, \cdots, \alpha_{J_{2}}^{2}\right)
\end{aligned}
$$

where $\alpha_{*}$ are the edges in each boundary listed in cyclic order.

Similar to the process illustrated in Section VI, we regard the whole division of $\Omega$ as a three-stage process. In the first stage, we consider the generation of the cutting paths connecting $\omega$ and $\omega^{1}$. As per the discussion in Section VI-C at this point there are only two cutting paths, generating two sub-cells. In the second stage, we place $\omega^{2}$ in each of the sub-cells. In either case, the sub-cell containing $\omega^{2}$ becomes a genus-one cell. Then it can be solved through further division. After creating other two cutting paths, $\Omega$ is transformed into three simply-connected sub-cells. In the third stage, all other cutting paths are created.

\section{B. Negligibility of Putting Inner Part on the Cutting Paths}

A special case may first appear to arise when placing $\omega^{2}$ within each sub-cell generated in the first stage intersecting the actual cutting path created (seen in blue in Fig. 8) Yet this is a case that requires no consideration given the equivalence shown in the same Figure. Since $\omega^{2}$ is not assumed unconnectable, if a cutting path lies on the existing topological edges, it prevents further connections (such as the one shown in red in Fig. 8), leading to higher cost. Recalling the result from Section VI-C there are only two cutting paths dividing $\Omega$ into two parts, so there are exactly two different places to put $\omega^{2}$, i.e., in either sub-cell created in the first stage.

\section{Complexity Analysis of Solving Genus-2 Cells}

When $\omega^{1}$ and $\omega^{2}$ are both disconnected, similar to 25 , we have

$$
\Phi_{00}\left(J_{1}, J_{2}, K\right)=2^{J_{1}}+2^{J_{2}}+2^{K}
$$




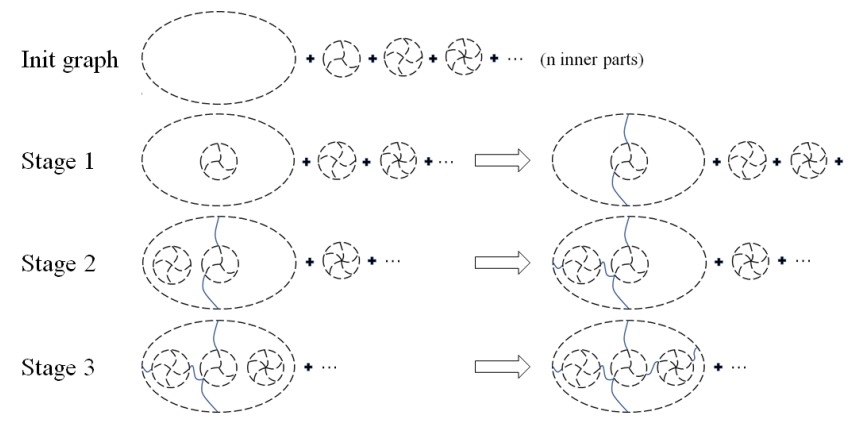

Fig. 9. Iterative transformation process of the genus- $n$ case.

If $\omega$ and $\omega^{1}$ are connected, but $\omega^{2}$ is disconnected,

$$
\Phi_{10}\left(J_{1}, J_{2}, K\right)=\Psi\left(J_{1}, K\right)+\Phi\left(J_{2}\right)
$$

exchanging the role of $\omega^{1}$ and $\omega^{2}$,

$$
\Phi_{01}\left(J_{1}, J_{2}, K\right)=\Psi\left(J_{2}, K\right)+\Phi\left(J_{1}\right)
$$

When both $\omega^{1}$ and $\omega^{2}$ are connected, the complexity can be calculated by considering $\omega^{2}$ being placed in different subcells separately. Using $\Xi_{1}$ for the situations that $\omega^{2}$ is placed in sub-cell 1 , and $\Xi_{2}$ for sub-cell 2 give rise to

$$
\begin{aligned}
& \Xi_{1}\left(J_{2} ; r_{1}, J_{1}, s, K\right)=\Psi\left(J_{2}, r_{1}+s\right) \Phi\left(J_{1}+K-r_{1}-s\right) \\
& \Xi_{0}\left(J_{2} ; r_{1}, J_{1}, s, K\right)=\Phi\left(r_{1}+s\right) \Psi\left(J_{2}, J_{1}+K-r_{1}-s\right)
\end{aligned}
$$

Going through all possible $r_{1}$ and $s$, we have

$$
\begin{aligned}
& \Psi\left(J_{1}, J_{2}, K\right)=\sum_{r_{1}=1}^{J_{1}-1} \sum_{s=1}^{K-1} \\
& r_{1} K\left[\Xi_{1}\left(J_{2} ; r_{1}, J_{1}, s, K\right)+\Xi_{0}\left(J_{2} ; r_{1}, J_{1}, s, K\right)\right]
\end{aligned}
$$

Summarising (30), 31), 32) and 35), the final result is compouned by

$$
\begin{aligned}
\Phi\left(J_{1}, J_{2}, K\right)= & \Phi_{00}\left(J_{1}, J_{2}, K\right)+\Phi_{01}\left(J_{1}, J_{2}, K\right) \\
& +\Phi_{10}\left(J_{1}, J_{2}, K\right)+\Psi\left(J_{1}, J_{2}, K\right)
\end{aligned}
$$

\section{Genus-n Cell Case}

Let $\Omega$ be a genus- $n$ cell, with its outer boundary denoted by $\omega$ and inner boundaries denoted by $\omega^{1}, \cdots, \omega^{n}$. The edges and their order in each boundary are given by 27] and

$$
\omega^{i}=\left(\alpha_{1}^{i}, \cdots, \alpha_{J_{i}}^{i}\right), \forall i=1, \cdots, n
$$

Again, we assume that all $\omega^{i}$ must be connected, or else the disconnected inner parts can be safely discarded, then the genus- $n$ problem becomes a lower-genus problem.

Similar to our discussion of the genus-two cell, the whole division of a genus- $n$ cell can be seen as an $(n+1)$-stage process. At each stage, $\omega^{i}$ is added to one of the sub-cells, transforming it into a genus-one sub-cell. Then, exactly two new cutting paths are required to transform this sub-cell again into two simply-connected sub-cells. After $n$ stages, $\Omega$ becomes an enssemble of simply-connected sub-cells. The process is illustrated by Fig. 9

\section{E. Complexity Analysis of Solving Genus-n Cells}

The number of different divisions can be classified based on whether each inner part is connected. For a genus- $n$ cell, define the subscript $T_{n}$ as a binary string with length $n$, where the digit sum of $T_{n}$ is $N\left(T_{n}\right)$. For example, $N\left(T_{n}\right)=0$ represents the case that none of the inner boundaries are connected. In this case,

$$
\Phi_{0 \cdots 0}\left(J_{1}, \cdots, J_{n}, K\right)=2^{J_{1}}+\cdots+2^{J_{n}}+2^{K}
$$

If $N\left(T_{n}\right)=1$, e.g., $\omega$ and $\omega^{1}$ are connected - and a genus$(n-1)$ structure is kept after the whole division - the number of divisions are

$$
\Phi_{10 \cdots 0}\left(J_{1}, \cdots, J_{n}, K\right)=\Psi\left(J_{1}, K\right)+2^{J_{2}}+\cdots+2^{J_{n}}
$$

There are $\left(C_{n}^{1}-1\right)$ terms with $N\left(T_{n}\right)=1$, e.g., $\Phi_{010 \cdots 0}$ to $\Phi_{0 \cdots 01}$, that can be calculated through exchanging the indices. Similarily, an example for $N\left(T_{n}\right)=2$ can be given by

$$
\Phi_{110 \cdots 0}\left(J_{1}, \cdots, J_{n}, K\right)=\Psi\left(J_{1}, J_{2}, K\right)+2^{J_{3}}+\cdots+2^{J_{n}}
$$

and $\left(C_{n}^{2}-1\right)$ terms can be calculated through exchanging the indices,

$$
\Phi_{1 \cdots 10}\left(J_{1}, \cdots, J_{n}, K\right)=\Psi\left(J_{1}, \cdots, J_{n-1}, K\right)+2^{J_{n}}
$$

with other $\left(C_{n}^{n-1}-1\right)$ similar terms satisfying $N\left(T_{n}\right)=n-1$.

By induction, all the above mentioned terms can be calculated except for the one satisfying $N\left(T_{n}\right)=n$. After the first stage, we can choose which sub-cell each $\omega^{i}, i=2, \cdots, n$ will eventually lie in, and $\Xi_{T_{n-1}}$ are used to consider each situation, with the $i$-th binary digit in $T_{n-1}$ representing that $\omega^{i}$ lies in sub-cell 1 or sub-cell 2 . Let $B(T, i)$ be the value of the $i$-th digit in $T$,

$$
B(T, i)=\frac{T}{2^{n-i}} \bmod 2
$$

then

$$
\begin{aligned}
& \Xi_{T_{n-1}}\left(J_{2}, \cdots, J_{n} ; r_{1}, J, s, K\right) \\
= & \Psi\left(J_{i_{2}}, \cdots, J_{i_{P}}, r_{1}+s\right) \Psi\left(J_{i_{P+1}}, \cdots, J_{i_{n}}, J+K-r_{1}-s\right)
\end{aligned}
$$

where $i_{2}, \cdots, i_{n}$ are specified by

$$
\left\{\begin{array}{l}
B\left(T_{n-1}, i_{j}\right)=1, \quad j=2, \cdots, P \\
B\left(T_{n-1}, i_{j}\right)=0, \quad j=P+1, \cdots, n .
\end{array}\right.
$$

It is easy to see that the order of $J_{i_{*}}$ does not effect the result in 43 . So

$$
\begin{aligned}
& \Psi\left(J_{1}, \cdots, J_{n}, K\right)=\sum_{r_{1}=1}^{J_{1}-1} \sum_{s=1}^{K-1} \\
& \quad\left(r_{1} K \sum_{T_{n-1}=0}^{2^{n-1}-1} \Xi_{T_{n-1}}\left(J_{2}, \cdots, J_{n} ; r_{1}, J, s, K\right)\right)
\end{aligned}
$$

Summarising the results from (38), 39), 40, ,41, ,45,

$$
\begin{aligned}
\Phi\left(J_{1}, \cdots, J_{n}, K\right)= & \sum_{T_{n}=0}^{2^{n}-2} \Phi_{T_{n}}\left(J_{1}, \cdots, J_{n}, K\right) \\
& +\Psi\left(J_{1}, \cdots, J_{n}, K\right)
\end{aligned}
$$




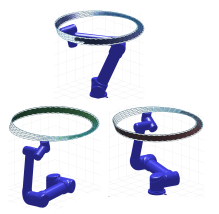

(a)

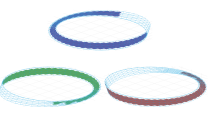

(b)

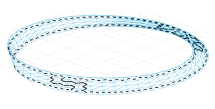

(c)

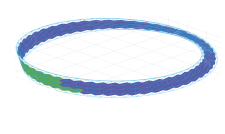

(d)
Fig. 10. Proposed NCPP applied to a Mobiüs strip (a) Examples of different configurations. (b) Coverable area of each the corresponding (colour) configuration. (c) Topological graph (d) One optimal solution requiring 1 liftoffs.

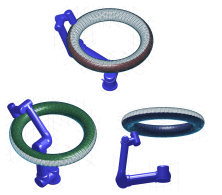

(a)

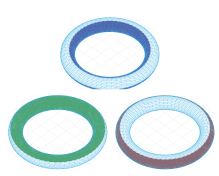

(b)

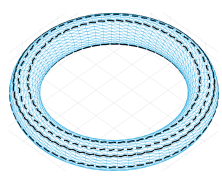

(c)

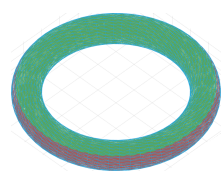

(d)
Fig. 11. Proposed NCPP applied to a closed surface ring(a) Examples of different configurations, where it becomes apparent that significant discontinuities will be inevitable without proper planning should the manipulator motion stretch in and out of the ring. (b) Continuous coverable area of three (colour) configurations, all of them being genus-one cells. (c) Topological graph generated. (d) One optimal solution, where only 1 lift-off is required.

\section{EXPERIMENTAL RESULTS}

Several representative simulation works have been implemented to validate the proposed algorithm on challenging arbitrarily-shaped objects.

Fig. 10 presents the solution of polishing a Mobiüs strip, whose surface is non-orientable. The manipulator is required to maintain the EE normal to the surface for proper operation simulating a contact task such as polishing. This is rather challenging in this case since the strip is twisted, hence the orientation of the normal vector varies over the full $2 \pi$ rads. However, the proposed algorithm is able to come up with an configuration mapping leading to an optimal solution where only 1 lift-off is required over the entire object.

Fig. 11 depicts the process of covering the surface of a "swimming" ring, another closed surface with no boundary. This is a particularly challenging case, it can be seen how the topological graph is formed by cells which are all multiplyconnected, yet the proposed algorithm is able to come up with an effective solution with a single configuration discontinuity to the NCPP problem.

The examples in Fig. 2, Fig. 12 and Fig. 13 illustrate the solutions of covering a "hilly terrain" with varying degrees of desirable manipulability [21]. For a given configuration (colour) cell, the manipulability is explicitly depicted brighter for increasing manipulability for the given configuration. The threshold varies from a minium of 0.06 (Fig. 2) to at least 0.10 (Fig. 12), up to at least 0.16, the maximum where a solution can be found for the object (in Fig. 13).

As one would expect, a sharp reduction on the reachable area is observed as the manipulability tightens (mainly due to the limited mobility of the wrist-flipped configurations, shown in the bottom two configurations in Fig. 12(a), causing large fluctuations in the structure of the resulting cells and topology graphs. When the threshold goes to 0.16 , most of the

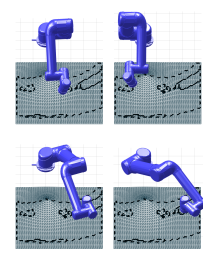

(a)

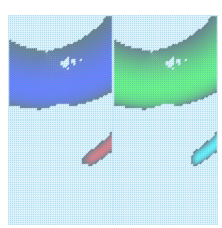

(b)

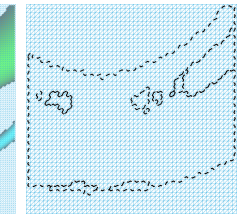

(c)

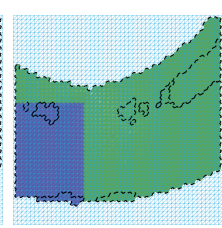

(d)
Fig. 12. (a) Examples of configurations belonging to different colours, where the threshold of manipulability at each point is set to $>0.1$. (b) Coverable area of the corresponding colours. We can see the wrist-flipped configurations can only cover reduced areas, and the configurations can no longer reach the area near the base of the manipulator (as compared with Fig. 2. where the minimum threshold is set to $>0.06$ ). (c) Topological graph. (d) An optimal solution with 1 lift-off.

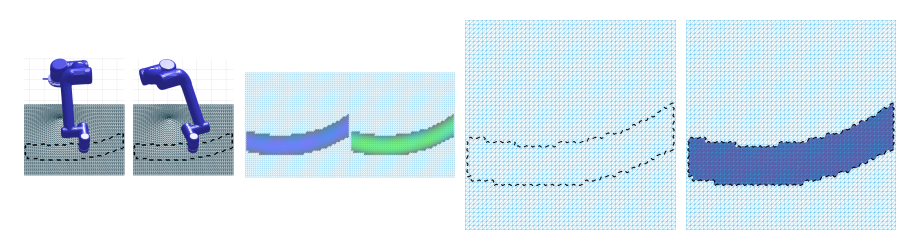

(a)

(b)

(c)

(d)

Fig. 13. Like Fig. 12 where the threshold of manipulability is set to $>0.16$.

kinematic-valid wrist-unflipped configurations are no longer valid, as depicted in Fig. 13 .

\section{CONCLUSION}

This paper has proposed a theoretical complete solution to the optimal non-revisting coverage task problem of arbitrarily shaped objects with a non-redundant manipulator. Given topological graphs of surface cells corresponding to feasible and continuous manipulator configurations, the scheme ensures optimality with respect to the number of surface discontinuities, and is able to deal with non simply-connected space topologies, which arise for complex objects and as the number of task constraints limit the set of feasible configurations.

In considering the generic solution to non simply-connected cells found in realistic environments, the proposed scheme effectively transforms the optimal CPP problem into an optimal colour designing problem of a topological configuration graph where a systematic solution for all genus cells is proposed. Through efficiently discarding equivalent and non-optimal cellular decompositions, the finiteness of divisions has been proven, and an upper bound in the number of divisions is calculated.

Challenging simulation scenarios suplemented by a video are provided to validate the proposed scheme.

\section{ACKNOWLEDGEMENT}

This work was supported by National Key R\&D Program of China (2018YFB1309300) National Nature Science Foundation of China (61903332, U1609210). The authors would like to thank Jin Gao for her careful examination on the formulae proposed in this paper. 


\section{REFERENCES}

[1] Ercan U. Acar, Howie Choset, Alfred A. Rizzi, Prasad N. Atkar, and Douglas Hull. Morse decompositions for coverage tasks. The International Journal of Robotics Research, 21(4):331-344, 2002. doi: 10.1177/ 027836402320556359. URL https://doi.org/10.1177/ 027836402320556359 .

[2] Prasad N Atkar, Howie Choset, and Alfred A Rizzi. Towards optimal coverage of 2-dimensional surfaces embedded in $\mathbb{R}^{3}$ : choice of start curve. In Proceedings of the 2003 IEEE/RSJ International Conference on Intelligent Robots and Systems, volume 4, pages 3581-3587. IEEE, 2003. doi: 10.1109/IROS.2003.1249711. URL https://doi.org/10.1109/IROS.2003.1249711.

[3] Prasad N Atkar, David C Conner, Aaron Greenfield, Howie Choset, and Alfred A Rizzi. Hierarchical segmentation of piecewise pseudoextruded surfaces for uniform coverage. IEEE Transactions on Automation Science and Engineering, 6(1):107-120, 2009. doi: 10.1109/TASE. 2008.916768. URL https://doi.org/10.1109/TASE.2008. 916768 .

[4] Chien-Chern Cheah, Sadao Kawamura, and Suguru Arimoto. Stability of hybrid position and force control for robotic manipulator with kinematics and dynamics uncertainties. Automatica, 39(5):847-855, 2003. doi: 10.1016/S0005-1098(03)00002-5. URL http://doi.org/10. 1016/S0005-1098(03)00002-5

[5] Howie Choset. Coverage for robotics-a survey of recent results. Annals of Mathematics and Artificial Intelligence, 31(1-4):113-126, 2001. doi: 10.1023/A:1016639210559. URL https://doi.org/10.1023/A:1016639210559

[6] Howie Choset, Ercan Acar, Alfred A Rizzi, and Jonathan Luntz. Exact cellular decompositions in terms of critical points of morse functions. In Proceedings of the 2000 IEEE International Conference on Robotics and Automation, volume 3, pages 2270-2277. IEEE, 2000. doi: 10.1109/ROBOT.2000.846365. URL http://doi.org/ 10.1109/ROBOT.2000.846365

[7] Enric Galceran and Marc Carreras. A survey on coverage path planning for robotics. Robotics and Autonomous Systems, 61(12):1258-1276, 2013. doi: 10.1016/j.robot. 2013.09.004. URL http://doi.org/10.1016/j.robot.2013. 09.004 .

[8] Ibrahim A Hameed, Anders la Cour-Harbo, and Ottar L Osen. Side-to-side $3 d$ coverage path planning approach for agricultural robots to minimize skip/overlap areas between swaths. Robotics and Autonomous Systems, 76: 36-45, 2016. doi: 10.1016/j.robot.2015.11.009. URL http://doi.org/10.1016/j.robot.2015.11.009.

[9] Dennis Heck, Alessandro Saccon, N Van De Wouw, and Henk Nijmeijer. Switched position-force tracking control of a manipulator interacting with a stiff environment. In Proceedings of the American Control Conference, pages 4832-4837. IEEE, 07 2015. doi: 10.1109/ACC. 2015.7172090. URL http://doi.org/10.1109/ACC.2015. 7172090 .

[10] W. H. Huang. Optimal line-sweep-based decomposi- tions for coverage algorithms. In Proceedings of the 2001 IEEE International Conference on Robotics and Automation, pages 27-32 vol.1. IEEE, 05 2001. doi: 10.1109/ROBOT.2001.932525. URL http://doi.org/10. 1109/ROBOT.2001.932525

[11] Steven M. LaValle. The Configuration Space, pages 105152. Cambridge University Press, 2006. doi: 10.1017/ CBO9780511546877.006. URL http://doi.org/10.1017/ CBO9780511546877.006

[12] Steven M LaValle and James J Kuffner Jr. Randomized kinodynamic planning. The International Journal of Robotics Research, 20(5):378-400, 2001. doi: 10. 1177/02783640122067453. URL http://doi.org/10.1177/ 02783640122067453

[13] Vladimir J Lumelsky, Snehasis Mukhopadhyay, and Kang Sun. Dynamic path planning in sensor-based terrain acquisition. IEEE Transactions on Robotics and Automation, 6(4):462-472, 1990. doi: 10.1109/70.59357. URL http://doi.org/10.1109/70.59357.

[14] Salehian Seyed Sina Mirrazavi and Billard Aude. A dynamical-system-based approach for controlling robotic manipulators during noncontact/contact transitions. IEEE Robotics \& Automation Letters, 3(4):2738-2745, 2018. doi: 10.1109/LRA.2018.2833142. URL http://doi.org/10. 1109/LRA.2018.2833142

[15] G. Oriolo and C. Mongillo. Motion planning for mobile manipulators along given end-effector paths. In Proceedings of the 2005 IEEE International Conference on Robotics and Automation, pages 2154-2160, 2005. doi: 10.1109/ROBOT.2005.1570432. URL http://doi.org/10. 1109/ROBOT.2005.1570432

[16] Fabian Paus, Peter Kaiser, Nikolaus Vahrenkamp, and Tamim Asfour. A combined approach for robot placement and coverage path planning for mobile manipulation. In Proceedings of the 2017 IEEE/RSJ International Conference on Intelligent Robots and Systems, pages 6285-6292. IEEE, 2017. doi: 10.1109/IROS. 2017.8206531. URL http://doi.org/10.1109/IROS.2017. 8206531

[17] George F. Simmons. Introduction to topology and modern analysis. The American Mathematical Monthly, 71 (4), 1964.

[18] J Ernesto Solanes, Luis Gracia, Pau Munozbenavent, Alicia Esparza, Jaime Valls Miro, and Josep Tornero. Adaptive robust control and admittance control for contact-driven robotic surface conditioning. Robotics and Computer-Integrated Manufacturing, 54:115-132, 2018. doi: 10.1016/j.rcim.2018.05.003. URL http://doi.org/10. 1016/j.rcim.2018.05.003.

[19] J. Ernesto Solanes, Luis Gracia, Pau Muoz-Benavent, Jaime Valls Miro, Carlos Perez-Vidal, and Josep Tornero. Robust hybrid position-force control for robotic surface polishing. Journal of Manufacturing Science and Engineering, 141(1), 11 2018. doi: 10.1115/1.4041836. URL http://doi.org/10.1115/1.4041836.

[20] Tong Yang, Jaime Valls Miro, Qianen Lai, Yue Wang, and Rong Xiong. Cellular decomposition for nonrepetitive coverage task with minimum discontinuities. 
IEEE/ASME Transactions on Mechatronics, 2020. doi: 10.1109/TMECH.2020.2992685. URL http://doi.org/10. 1109/TMECH.2020.2992685.

[21] Tsuneo Yoshikawa. Translational and rotational manipulability of robotic manipulators. In Proceedings of the 1991 International Conference on Industrial Electronics, Control and Instrumentation, pages 1170-1175, 1991. doi: 10.1109/IECON.1991.239275. URL http://doi.org/ 10.1109/IECON.1991.239275. 